http://jmscr.igmpublication.org/home/ ISSN (e)-2347-176x ISSN (p) 2455-0450 crossref DOI: https://dx.doi.org/10.18535/jmscr/v7i8.14

\title{
Mental Health Assessment of School going Adolescents (11-17 years) in Relation to Mother Education and Employment in Jaipur, Rajasthan
}

\author{
Authors \\ Rekha Shekhawat ${ }^{1}$, Nikita Sharma ${ }^{2 *}$, Vikram Singh Sodhas $^{3}$, Amita Kashyap ${ }^{4}$ \\ SMS Medical College, Jaipur (Rajasthan), India \\ *Corresponding Author \\ Nikita Sharma \\ SMS Medical College, Jaipur (Rajasthan), India
}

\begin{abstract}
Introduction: The adolescents comprise one fifth Indian population and mothers play a key role in their physical, mental and social development. So this study was done to assess mental health problems in school going adolescents and to find association with their mother's education and employment.

Methodology: This was a cross-sectional study on four governments and four private school going adolescents (11-17 years) studying in 6th to 12th class in Jaipur, Rajasthan. The simple random sampling was done for selection of schools and students. The self reported version of strength \& difficulty questionnaire (SDQ) and socio-demographic proforma were used as study tool. Microsoft-excel and primer software were used for data analysis.

Results: the $8.35 \%$ and $14.19 \%$ students had got abnormal and borderline total difficulty score respectively. The most prevalent problem was conduct (12\%) followed by Emotional (10\%), Peer (7\%), Hyperactivity (6\%) and Prosocial Problem (5\%). The mental problems were higher in those mother were working than non-working/housewives (10 vs 8\%), though insignificant. But mother education was significantly associated with mental problems ( $p$ value $<0.05$ ).

Conclusion: mental problems were found in $8.35 \%$ adolescents that need further evaluation and treatment by psychiatrist. Mother education is important for good mental health of their child.
\end{abstract}

Keywords: $S D Q$, Total difficulty score, borderline, adolescent.

\section{Introduction}

Adolescent word is derived from Latin term "adolescere" which means "to grow up". ${ }^{[1]}$ It is a transitional period between childhood and adulthood. Every one fifth population is adolescent in India. ${ }^{[2]}$ They are the building-blocks of the nation, forming a major demographic and economic force. The adolescents have to face many health problems like mental health problems, early pregnancy and childbirth, human immunodeficiency virus/sexually transmitted infection and other infectious diseases, violence, unintentional injuries, malnutrition and substance abuse. ${ }^{[3]}$

The adolescent mental health problems cause significant educational, social and emotional burden not only to themselves but also to parents and families ultimately to country. The twenty percent of the world's adolescent population have a mental health or behavioural problem. ${ }^{[4]}$ In India it is estimated $6.33 \%$ to $46 \%$ according to school based studies. $^{[5]-[6]}$ 
Adolescents spend majority of their time in school other than home. The school teachers can be helpful in identifying mental health problems of adolescents $^{[7]}$ but they are not well trained for this. Also the primary healthcare providers may not be managing these problems/disorders in their routine busy practices because the lack of time and patience required in identifying these problems. So in these situations importance of screening tools or procedures have been increased.

The mother as one of the two pillars of a family plays important role in rearing children, taking care of family and establishing a friendly emotional atmosphere. But in present scenario greater changes had occur in the women's individual, family, and social roles. It was seen that in the early years of a child's life is linked to worse cognitive and behavioural outcomes particularly among more advantaged mothers. ${ }^{[8]}$

With this in mind present study was planned to assess mental health of school going adolescents (11-17 years) in Jaipur, Rajasthan and to find out association of mental health problems with mother education and employment (working/non-working)

\section{Methodology}

This cross-sectional study was done in Jaipur, Rajasthan during one year of period on students of age group 11-17 years of randomly selected schools of Jaipur city.

Inclusion Criteria: Students of 11 to 17 years studying in Government and Private Senior secondary schools ( $\geq 500$ students) of Jaipur city and student present on the day of study.

Exclusion Criteria: Those whose school authorities did not give consent and those who are absent or ill. The sample size required is 794 at $95 \%$ confidence and $2 \%$ absolute error to verify the expected minimum $8.7 \%$ of abnormal SDQ score. ${ }^{[9]}$ All schools were categorized into government and private schools in each zone of the city (East, West, North, and South zone.) One government and one private school from all four zone was randomly selected using simple random sampling technique i.e. 8 schools in total. The 126 children from each school were randomly selected; hence 18 randomly selected children from each class (6th to 12th) were included. All 1008 randomly selected students were distributed Socio-demographic and SDQ questionnaire, after explaining them the purpose of study and promise of anonymity. Out of 1008 students 994 students were filled the questionnaire completely.

The following tools were used in this study Sociodemographic Proforma: pretested and structured

Strength and difficulties questionnaire: The SDQ is a user-friendly screening questionnaire (validated), which can be used to assess behavioural problems and mental health disorders. In study selfreport version suitable for adolescents aged around 11-17, depending on their level of understanding and literacy was used. The SDQ ask about 25 attributes that are divided between 5 scales (five items in each scale): 1) Emotional symptoms scale 2) Conduct problems scale 3) Hyperactivity scale 4) Peer problems scale 5) Prosocial scale By adding the scores of Emotional, Conduct, Hyperactivity and Peer Problems scale, the Total Difficulty score or the Mental Health status was generated. An abnormal score on total difficulty score was taken as mental health problems. The score of Pro-social Behaviour was not taken into account for this purpose. Total scores are only calculated if at least three of the five items have been completed. Interpretation of SDQ scoring values:

$\begin{array}{lccc}\text { The SDQ scoring: } & \text { Normal } & \text { Borderline } & \text { Abnormal } \\ \text { Total difficulty score } & 0-15 & 16-19 & 20-40 \\ \text { Emotional symptoms score } 0-5 & 6 & 7-10 \\ \text { Conduct problems score } & 0-3 & 4 & 5-10 \\ \text { Hyperactivity score } & 0-5 & 6 & 7-10 \\ \text { Peer problems score } & 0-3 & 4-5 & 6-10 \\ \text { Prosocial score } & 6-10 & 5 & 0-4\end{array}$

Statistical Analysis: The collected data were classified, tabulated and analyzed as per the aims and objectives. The data obtained were analyzed by using both descriptive and inferential statistics. The chi square test was used to find out association and $\mathrm{p} \leq 0.05$ was considered statistically significant. The "Microsoft Excel" and "Primer" were used for data analysis. Ethical consideration 
This study was reviewed and approved by the SMS Medical College, Jaipur Ethical Committee. Permission to collect information was taken from District Education officer and respective authorities of the selected schools.

\section{Results}

Out of total 994 students (those filled questionnaire completely) majority were females $(51 \%)$ and were in age group $15-17(45 \%)$. The mothers of majority were illiterate $(45 \%)$ and non working; housewife (84\%). (Table 1)

The mental health problem was found in eight percent adolescents. Conduct Problem was the most prevalent among adolescent (12\%), followed by Emotional Problem (10\%), Peer Problems (7\%), Hyperactivity (6\%) and Prosocial Problem (5\%). (Table 2).
The poor mental health (abnormal and borderline) was significantly highest in those belonged to illiterate mothers $(24 \%)$. The proportion of abnormal and borderline in emotional problem were also highest in those belonged to illiterate mothers $920 \%$ ) and peer problem was also highest in those mother were illiterate (7\%). (Table 3 ).

The mental health problem was higher in those belonged to working mothers than nonworking mothers/housewives (10 vs $8 \%)$, though insignificant. Likewise emotional (12\%) and hyperactivity problems (10\%) were more in those belonged to working mothers while conduct (12\%), peer $(8 \%)$ and prosocial problems $(6 \%)$ were more in those belonged to nonworking mothers (Table 4)

Table 1: Demographic Characteristic of adolescents (N=994)

\begin{tabular}{|lccc|}
\hline Variable & Subgroup & Total (N) & Percentage (\%) \\
Age groups (years) & $\mathbf{1 1 - 1 2}$ & 253 & 25.5 \\
& $\mathbf{1 3 - 1 4}$ & 292 & 29.4 \\
Sex & $\mathbf{1 5 - 1 7}$ & 449 & 45.2 \\
& Male & 485 & 48.8 \\
Mother education & Female & 509 & 51.2 \\
& Illiterate & 451 & 45.4 \\
& Primary & 61 & 6.1 \\
& Secondary & 292 & 29.4 \\
Mother working status & Higher secondary & 190 & 19.1 \\
& Working & 156 & 15.7 \\
& Non-working & 838 & 84.3 \\
\hline
\end{tabular}

Table 2: Distribution of mental health problems in adolescents

\begin{tabular}{|lccc|}
\hline Categories & Normal(\%) & Borderline(\%) & Abnormal(\%) \\
Total difficulty score & $770(77.46)$ & $141(14.19)$ & $83(8.35)$ \\
Emotional problems & $824(82.90)$ & $67(6.74)$ & $103(10.36)$ \\
Conduct problems & $766(77.06)$ & $112(11.27)$ & $116(11.67)$ \\
Hyperactivity problems & $846(84.11)$ & $86(8.65)$ & $62(6.24)$ \\
Peer problems & $714(71.83)$ & $207(20.82)$ & $73(7.34)$ \\
Prosocial behaviour problems & $877(88.23)$ & $64(6.44)$ & $53(5.33)$ \\
Total impact score & $777(78.16)$ & $80(8.04)$ & $137(13.78)$ \\
\hline
\end{tabular}


Table 3: Association of mother education with mental health problems

\begin{tabular}{|c|c|c|c|c|c|c|}
\hline Category & $\begin{array}{c}\text { Illiterate } \\
\mathbf{N}=451\end{array}$ & $\begin{array}{c}\text { Primary } \\
N=61\end{array}$ & $\begin{array}{c}\text { Secondary } \\
\mathrm{N}=292\end{array}$ & $\begin{array}{c}\geq \text { Higher } \\
\text { secondary } \\
\mathbf{N}=190\end{array}$ & Total $=994$ & $\begin{array}{c}\text { Chi- } \\
\text { square } \\
\text { P value }\end{array}$ \\
\hline \multicolumn{7}{|c|}{ Tptal difficulty score (Mental Health) \% } \\
\hline Normal & $343(76.1)$ & $53(86.9)$ & 236(80.8) & 138(72.6) & 770 & 28.439 \\
\hline Borderline & 73(16.2) & $2(3.3)$ & $44(15.1)$ & $22(7.6)$ & 141 & $<0.001$ \\
\hline Abnormal & $35(7.8)$ & $6(9.8)$ & $12(4.1)$ & $30(10.3)$ & 83 & \\
\hline \multicolumn{7}{|c|}{ Emotional Problems(\%) } \\
\hline Normal & $359(79.6)$ & $54(88.5)$ & 252(86.3) & 159(83.7) & 824 & 18.805 \\
\hline Borderline & $35(7.8)$ & $7(11.5)$ & $17(5.8)$ & $7(3.7)$ & 67 & 0.005 \\
\hline Abnormal & $57(12.6)$ & 0 & $22(7.5)$ & 24(12.6) & 103 & \\
\hline \multicolumn{7}{|c|}{ Conduct problems $(\%)$} \\
\hline Normal & $362(80.3)$ & 47(77.0) & 221(75.4) & 136(71.6) & 766 & 12.681 \\
\hline Borderline & 49(10.9) & $10(16.4)$ & 29(9.9) & 24(12.6) & 112 & 0.048 \\
\hline Abnormal & $40(8.9)$ & $4(6.6)$ & 42(14.4) & $30(15.8)$ & 116 & \\
\hline \multicolumn{7}{|c|}{ Hyperactivity problems(\%) } \\
\hline Normal & $379(84.0)$ & $50(82.0)$ & 258(88.4) & $159(83.7)$ & 846 & 4.463 \\
\hline Borderline & $43(9.5)$ & $7(11.5)$ & $20(6.8)$ & $16(8.4)$ & 86 & 0.614 \\
\hline Abnormal & $29(6.4)$ & $4(6.6)$ & $14(4.8)$ & $15(7.9)$ & 62 & \\
\hline \multicolumn{7}{|c|}{ Peer problems $(\%)$} \\
\hline Normal & $314(69.6)$ & $40(65.6)$ & 217(74.3) & 143(75.3) & 714 & 13.946 \\
\hline Borderline & $104(23.1)$ & 17(27.9) & $60(20.7)$ & 26(13.7) & 207 & 0.030 \\
\hline Abnormal & $33(7.3)$ & $4(6.6)$ & $15(5.1)$ & $21(6.8)$ & 73 & \\
\hline \multicolumn{7}{|c|}{ Prosocial behaviour problems(\%) } \\
\hline Normal & $397(88.0)$ & $56(91.8)$ & $260(89.0)$ & 164(86.3) & 877 & 2.125 \\
\hline Borderline & $31(8.8)$ & $3(4.9)$ & $17(5.8)$ & $13(6.8)$ & 64 & 0.908 \\
\hline Abnormal & $23(5.1)$ & $2(3.3)$ & $15(5.1)$ & $13(6.8)$ & 53 & \\
\hline
\end{tabular}

Table 4: Association of Mental health problems with employment status of mothers

\begin{tabular}{|lcccc|}
\hline Category & $\begin{array}{c}\text { Working mother } \\
\text { N=156 }\end{array}$ & $\begin{array}{c}\text { Non-working } \\
\text { mother N=838 }\end{array}$ & Total=994 & $\begin{array}{c}\text { Chi-square } \\
\text { P value }\end{array}$ \\
Total difficulty score(Mental health) $\%$ & & & \\
Normal & $127(81.4)$ & $643(76.7)$ & 770 & 4.269 \\
Borderline & $14(8.9)$ & $127(15.2)$ & 141 & 0.118 \\
Abnormal & $15(9.6)$ & $68(8.1)$ & 83 & \\
Emotional problems(\%) & $126(80.8)$ & $698(83.3)$ & 824 & 0.717 \\
Normal & $11(7.1)$ & $56(6.7)$ & 67 & 0.699 \\
Borderline & $19(12.2)$ & $84(10)$ & 103 & \\
Abnormal & & & & \\
Conduct problems(\%) & $125(80.1)$ & $641(76.5)$ & 766 & 4.004 \\
Normal & $20(12.8)$ & $92(11)$ & 112 & 0.135 \\
Borderline & $11(7.1)$ & $105(12.5)$ & 116 & \\
Abnormal & & & \\
Hyperactivity problems(\%) & $130(83.3)$ & $716(85.5)$ & 846 & 4.002 \\
Normal & $11(7.1)$ & $75(8.9)$ & 86 & 0.135 \\
Borderline & $15(9.7)$ & $47(5.6)$ & 62 & \\
Abnormal & & & & \\
Peer problems(\%) & $124(79.5)$ & $590(70.4)$ & 714 & 5.745 \\
Normal & $22(14.1)$ & $185(22.1)$ & 207 & 0.057 \\
Borderline & $10(6.4)$ & $63(7.5)$ & 73 & \\
Abnormal & & & \\
Pro-social problems(\%) & $138(88.5)$ & $739(88.2)$ & 877 & 1.219 \\
Normal & $12(7.7)$ & $52(6.2)$ & 64 & 0.544 \\
Borderline & $6(3.8)$ & $47(5.6)$ & 53 & \\
Abnormal & & & \\
\hline
\end{tabular}

\section{Discussion}

This cross-sectional study provides a generalized assessment of mental health status of school-going adolescents aged 11-17 years in Jaipur, Rajasthan by using SDQ screening tool and to find out any relation of mental health problems of adolescents 
with their mothers education and employment (working or housewife). This study concluded that $8 \%$ adolescents had got abnormal total difficulty score which means they have defiantly mental health problems/disorders and they need further evaluation and appropriate treatment and counselling. Furthermore $14 \%$ adolescents were on borderline score; they should also take care by parents and teachers for prevention of future adverse consequences. The conduct problem (12\%) was highest and prosocial (5\%) was least problem in adolescents. The other Indian studies ${ }^{[9]-[12]}$ also estimated prevalence of abnormal mental health ranging from 9 to $15 \%$. These differences may be due to difference in study area, age group, screening tool used, source of information, time interval and case definition included in the study.

This study showed a significant association of mother education with mental health problems. The study concluded that proportion of borderline and abnormal adolescents (24\%) on total difficulty and emotional problems (20\%) were highest in those belonged to illiterate mother. Peer problems were also highest in those belonged to illiterate mother. This may be explained by educated mother have better knowledge with respect to child rearing and one more factor. Rahi $\mathrm{M}$ et $\mathrm{al}^{[13]}$ and Banstola RS et $\mathrm{al}^{[14]}$ also concluded that whose mothers were illiterate more likely to have psychosocial problem significantly.

This study was found no significant association between employment status mothers and mental health problems although more prevalence was found in those mothers were working (10\% vs $8 \%$ ). This may be because maternal employment not only influences child outcomes but also has effects on the family processes that mediate child outcomes, namely: the psychological well-being of the parents, their marital relationship, the father's role, and parent-child interactions. Adverse family climate is also a negative contributor to children's mental health. The emotional and hyperactivity problems were more in those both parents were working while conduct, peer and prosocial problems were more in those mothers were non-working (Housewife).
Seenivasan $\mathrm{P}$ et $\mathrm{al}^{[12]}$ and Agrawal $\mathrm{S}$ et al ${ }^{[10]}$ studies found similar association but significant and in all mental problems but Arman A et al ${ }^{[14]}$ study found that psychiatric problems were 2.44 folds higher in housekeeper mother than working mothers.

\section{Conclusion}

This study provides a snapshot of vulnerability among school going adolescents of Jaipur city, Rajasthan; proportion of borderline and abnormal adolescents was more than one fifth on total difficulty score. So they need earliest clinician diagnosis and appropriate treatment or counselling. Maternal education has major impact on mental health of their children.

\section{Recommendation}

The teachers at the school level should be trained for early detection of poor mental health and life skills education should be integrated in schools across the curriculum. There should be identified psychologist for each school to refer students in need for counselling and treatment.

\section{Limitation}

This study was based on self reported behaviour only; clinical indices could not be used to confirm this self-report measure. This study was done on urban schools only; not included rural adolescents; whose perception may be different.

Funding: No funding sources

Conflict of interest: None declared

Ethical approval: The study was approved by the Institutional Ethics Committee

\section{References}

1. Macmillan PL. Macmillan Dictionary for Students, 1st edition. New York: Simon \& Schuster Books for Young Readers; 1981.p 14.

2. Centers for Disease Control and Prevention. Youth Risk Behavior Surveillance; United States-2011,61(4).United states;2012.p53 
3. WHO: Maternal, newborn, child and adolescent health. Adolescent health epidemiology. Available at: http://www.who.int/maternal_child_adolesce nt/epidemiology/adolescence/en/ .Accessed on 28 September2018

4. Kalaiyarasan M, Solomon MD. Mental health in adolescence. IJRANSS 2014;2(8): 27-32.

5. Cholakottil A, Kazhungil F, Koyamu AM. Prevalence and Pattern of Psychiatric Disorders in School Going Adolescents. The International Journal of Indian Psychology; 4(3):124-8

6. Malhotra S, Kohli A, Kapoor M, Pradhan B. Incidence of childhood psychiatric disorders in India. Indian J Psychiatry 2009; 51:101-7

7. Seenivasan P, Kumar CP. A comparison of mental health of urban Indian adolescents among working and non working mothers. Ao CH 2014;2(2):39-43.

8. Malhotra S, Kohli A, Kapoor M, Pradhan B. Incidence of childhood psychiatric disorders in India. Indian J Psychiatry 2009; 51:101-7

9. Reddy B K, Biswas A, Rao H. Assessment Of Mental Health Of Indian Adolescents Studying In Urban Schools. MJPCH 2011;5(17):1-6.

10. Agrawal S, Shah R, Meena RK, Gaur K. Psychiatric Wellness among adolescent school going children of Jaipur City. Int $\mathbf{J}$ Multispecialty Journal 2015;1(5):7-14.

11. Bhola P, Sathyanarayanan V, Rekha DP, Daniel S, Thomas T. Assessment of selfreported emotional and behavioural difficulties among pre-university college students in Bangalore, India. Indian $\mathbf{J}$ Community Med 2016;41(2): 146-50.

12. Seenivasan P, Kumar CP. A comparison of mental health of urban Indian adolescents among working and non working mothers. AoCH 2014;2(2):39-43.
13. Rahi M, Kumawat AP, Garg S, Singh MM. Socio-demographic co-relates of psychiatric disorders.Indian J pediatr 2005;72:395-7

14. Arman S, Keypour M, Maracy, Attari A. Epidemiological Study of Youth Mental Health Using Strengths and Difficulties Questionnaire (SDQ). Iran Red Crescent Med J 2012;14(6):371-5. 